\title{
Effect of starch on the mechanical and in vitro properties of collagen- hydroxyapatite sponges for applications in dentistry
}

\author{
Ana B. Castro-Ceseña ${ }^{a 1^{*}}$, Tanya A. Camacho-Villegas ${ }^{\mathrm{b} 2}$, Pavel H. Lugo-Fabres ${ }^{\mathrm{b}}$, Ekaterina \\ E. Novitskaya ${ }^{c}$, Joanna McKittrick ${ }^{a, c}$, Alexei Licea-Navarro ${ }^{b}$ \\ ${ }^{a}$ Materials Science and Engineering Program, University of California, San Diego, La Jolla, CA \\ 92093, USA. \\ ${ }^{b}$ Biomedical Innovation Department. Centro de Investigación Científica y de Educación Superior \\ de Ensenada (CICESE), Ensenada, Baja California, Carretera Ensenada-Tijuana No. 3918, \\ Zona Playitas, C.P. 22860, Ensenada, Baja California, México. \\ ${ }^{c}$ Department of Mechanical and Aerospace Engineering, University of California, San Diego, La \\ Jolla, CA 92093, USA. \\ ${ }^{1}$ Present address: CONACYT Research Fellow - Centro de Investigación y Desarrollo \\ Tecnológico en Electroquímica, Subsede Tijuana (CIDETEQ) \\ ${ }^{2}$ Present address: CONACYT Research Fellow - Centro de Investigación y Asistencia en \\ Tecnología y Diseño del Estado de Jalisco, A.C. (CIATEJ)
}

Authors:

Tanya A. Camacho-Villegas tcamacho@ciatej.mx,

Pavel H. Lugo-Fabres: plugo@cicese.edu.mx,

Ekaterina E. Novitskaya: eevdokim@ucsd.edu,

Joanna McKittrick: imckittrick@eng.ucsd.edu

Alexei Licea-Navarro: alicea@cicese.mx

${ }^{*}$ Corresponding autor:

Ana B. Castro-Ceseña: acastro@cideteq.mx

Tel: +526646602054 


\begin{abstract}
This study sought to improve the mechanical and blood-absorbing properties of collagen sponges, while keeping them compressible, by incorporating blended hydroxyapatite (HA)starch. Results were compared with CollaPlug ${ }^{\circledR}$ (pure collagen). The elastic modulus increased from $1.5 \pm 0.2 \mathrm{kPa}$ for CollaPlug® to $49 \pm 8 \mathrm{kPa}$ for sponges of composition 1:4:10 (collagen:HA:starch, by weight). The modified microstructure and surface area provided by the starch granules on the sponges improved cell viability. Sponges with composition 1:4:10 maintained their blood-clotting capability with almost no change from 5 to 15 min after contact with blood, while CollaPlug ${ }^{\circledR}$ diminished to about half its capacity to absorb blood and form clots. Incorporation of HA-starch into the sponges with composition of 1:4:10, increased the elastic modulus of the collagen-HA sponges, making them more structurally robust. The viability of cells and the blood-clotting capability increased with starch incorporation.
\end{abstract}

Keywords: Starch, collagen, sponge, dental filler, blood-clotting, CollaPlug® 


\section{Introduction}

Spongeous scaffolds have several applications in dentistry, one of them as dental fillers (Araujo-Pires et al., 2015; Go, Teh, Tan, Zhang \& Teo, 2015). After tooth extraction, reduction of the alveolar ridge occurs as a natural physiological response during socket healing process (Hansson \& Halldin, 2012; Leblebicioglu et al., 2013). However, preservation of the alveola is necessary when placement of a dental implant is chosen as next step (Araujo-Pires et al., 2015; Go et al., 2015; Wang \& Lang, 2012). When dental fillers or dental plugs are used after tooth extraction, vertical and horizontal dimensions of the alveolar ridge are significantly less contracted (Thalmair, Fickl, Schneider, Hinze \& Wachtel, 2013; Vignoletti et al., 2012). Sponges are also used as internal matrices to act as barriers to prevent overextension of endodontic sealers (Zou, Liu, Yin, Li \& Xie, 2008), which in turn may result in injure of the inferior alveolar nerve, for example (López-López, Estrugo-Devesa, Jané-Salas \& Segura-Egea, 2012). Materials for such applications should be easy to process and be capable to stop bleeding or hemorrhage by promoting blood clotting (hemostasis) (Chang, Huang, Yang, Kuo \& Lee, 2012; Soares et al., 2015; Zou et al., 2008). Thus, sponges are considered optimal not only to maintain the dental cavity after tooth extraction (Kim, Yun, Lee, Ahn \& Kim, 2011; Serino, Biancu, lezzi \& Piatelli, 2003) by reducing alveolar bone resorption (Serino et al., 2003), but also as an internal matrix when placed through furcation perforations (Zou et al., 2008). Considering these characteristics, collagen sponges have been used as wound dressings for dental applications (Ohno et al., 2004; Taira, Sasaki, Saitoh, Nezu \& Araki, 2006), among others.

A sponge made of collagen is inherently biocompatible, and is also highly porous, a requirement to allow cell infiltration and exchange of oxygen and nutrients (Hiraoka, Kimura, Ueda \& Tabata, 2003). For example, CollaPlug ${ }^{\circledR}$ is a commercial bio-absorbable sponge made of type I collagen extracted from bovine Achilles tendon (Gruber, Lesli, Ingram, Norton \& Hanley, 2004) used for socket augmentation at the time of tooth extraction to reduce alveolar bone loss (Wang \& Tsao, 2007). However, despite collagen is considered the ideal framework for porous and biocompatible scaffolds, it has the disadvantage of quick degradation (Chang, et al., 2012) and weak mechanical properties (Yuniki, Ikoma \& Tanaka, 2010), as a result CollaPlug $®$ may not be suitable to protect against overextension when used as an internal matrix, together with dental sealers, to repair furcation perforations since is not pressure resistant (Zou et al., 2008). Therefore, for other applications such as bone graft and tissue engineering, collagen scaffolds have commonly been reinforced with hydroxyapatite (HA) (Kane \& Roeder, 2012; Kane et al., 2015; Yunoki et al., 2006), a biocompatible ceramic that increases not only the mechanical properties but also the osteoconductivity of the collagen scaffolds 
(Rodríguez et al., 2003; Wei \& Ma, 2004). However, it should be considered that even though the compression response of materials for applications in dentistry needs to be improved, it has to be in a way that scaffolds continue being compressible and capable of promoting hemostasis. Starch, a carbohydrate formed by a combination of linear (amylose, 20-30\%) and branched polymers (amylopectin, 70-80\%) (Nair \& Laurencin, 2005), is intrinsically biocompatible, biodegradable, water soluble and readily available. It has been used for fabrication of starchbased scaffolds for tissue engineering (Salgado, et al., 2002; Salgado, Coutinho \& Reis, 2004; Salgado, Figueiredo, Coutinho \& Reiss, 2005) and drug delivery (Shibata, Nishumura, Naruhashi, Nakao \& Miura, 2010). Sponges made of starch maintain their shape after absorbing liquid (Bice, MacMasters \& Hilbert, 1944), and have been used as absorbable hemostatic agents (Korchin, 1956). Based on this, we hypothesize that if sponges are prepared with collagen, HA and starch; then collagen would provide a framework for the sponges, HA would add stiffness, while the incorporation of starch will keep them compressible and bloodabsorbing. Therefore, the combination of HA-starch will lead to hemostatic sponges more structurally robust, which are important characteristics of hemostatic and space filler agents for dental surgery.

The present study sought to improve the mechanical and blood-absorbing properties of collagen sponges, while keeping them compressible, by incorporating blended HA and starch into the scaffolds. The elastic modulus, cell proliferation, and the blood-clotting properties of the sponges were investigated and compared with commercial product, CollaPlug®.

\section{Materials and methods}

\subsection{Raw materials and reagents}

The starch used to fabricate the sponges was food grade ( $94 \%$ cornstarch) from Unilever, México, and was used in as received conditions. Bovine femur bone was purchased from a local butcher (San Diego, California, US). Bone was demineralized using EDTA tetrasodium salt, obtained from Spectrum. The isolated collagen was dissolved into acetic acid, glacial, from Fisher Scientific (ACS grade) and compared with type I collagen from calf skin, purchased from Sigma-Aldrich, while the fabricated sponges were compared with CollaPlug ${ }^{\circledR}$ (Zimmer Dental, Indiana, US). Sintered hydroxyapatite (HA) (2-3 $\mu \mathrm{m}$ particle size) was obtained from TransTech. Mouse fibroblast L929 cells were gotten from the American Type Culture Collection (ATCC, CCL-1). RPMI 1640 medium, fetal bovine serum, antibiotic/antimicotic solution, trypsin/EDTA and Hanks' Balanced Salt Solution (HBSS) were purchased from GIBCO. Cell Titer 96 Aqueous One Solution was obtained from PROMEGA. 


\subsection{Collagen isolation}

The collagen used in this study was obtained by demineralization of cortical bovine femur bone using $0.1 \mathrm{M}$ EDTA at $20^{\circ} \mathrm{C}$. Bone demineralization was confirmed by measuring the $\mathrm{PO}_{4}{ }^{3-}-$ $P$ released into EDTA solution (Fig. 1a) using an UV/Vis spectrophotometer as described in detail elsewhere (Castro-Ceseña, Novitscaya, Phadke, Varghese \& McKittrick, 2013). Briefly, the cleaned cortical bovine bone was cut into pieces $\left(2 \mathrm{~cm}^{3}\right)$ and powdered using a mortar and pestle before immersed into EDTA solution. Demineralization was done for 9 days; while EDTA was refreshed every day. Quality of the isolated collagen (partially soluble) was verified by sodium dodecyl sulfate poly-acrylamide gel electrophoresis (SDS-PAGE) (Fig. 1b) (CastroCeseña et al., 2013). In this work, soluble collagen was obtained after solubilization of the demineralized powdered bone into $0.1 \mathrm{M}$ acetic acid, and then concentrating by freeze-drying (Labconco Freeze zone, 1L), referred to herein as acetic acid-solubilized collagen.

a

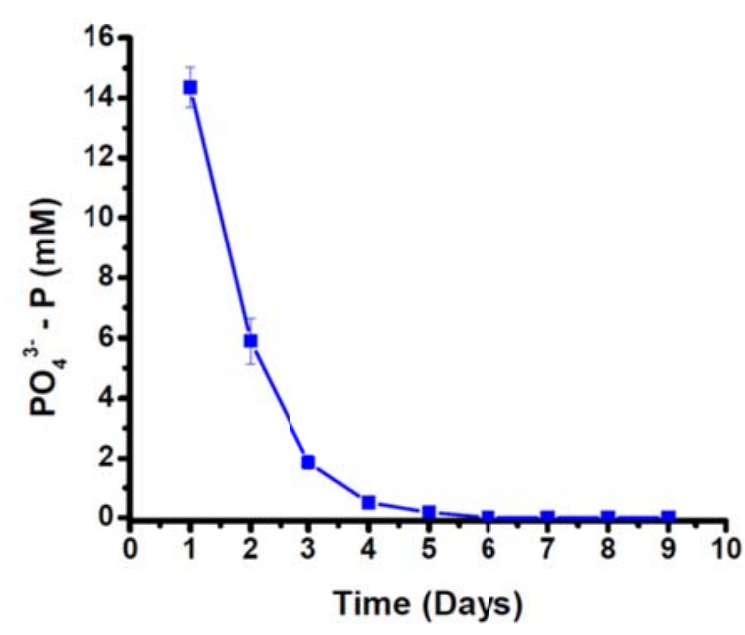

b

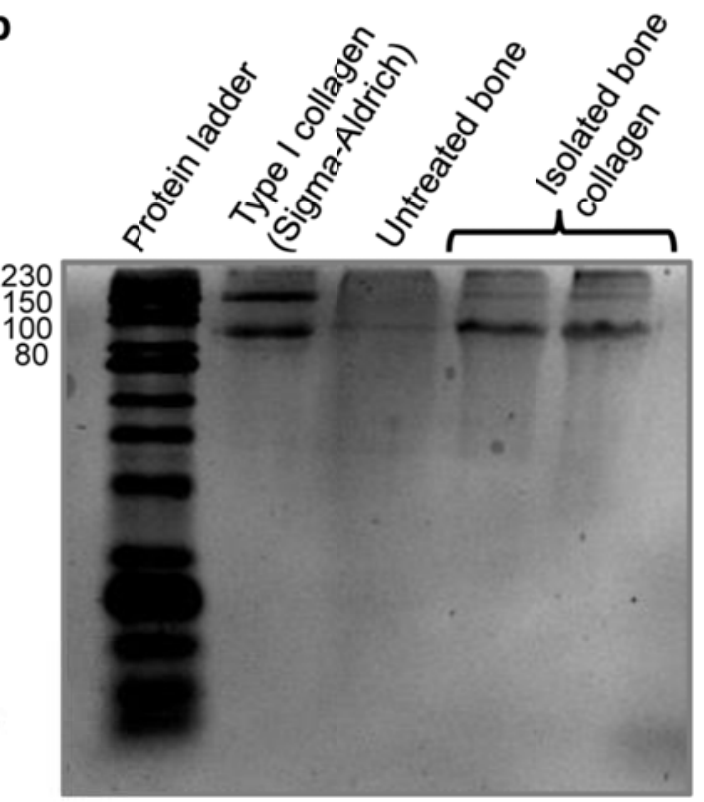

Fig. 1. Isolation of partially soluble collagen from bovine femur bone. (a) Confirmation of bone demineralization by measurement of $\mathrm{PO}_{4}{ }^{3-}-\mathrm{P}$ released into demineralization solution. (b) SDSPAGE of the partially soluble isolated collagen from bovine femur bone compared with commercial type I collagen and untreated bone. SDS-PAGE shows that the isolated collagen was not hydrolyzed. Modified from Castro-Ceseña, Novitscaya, Phadke, Varghese \& McKittrick, 2013. 


\subsection{Fabrication of the sponges}

All of the sponges were prepared using a concentration of $20 \mathrm{mg} / \mathrm{mL}$ of the acetic acidsolubilized collagen (Section 2.2) and $80 \mathrm{mg} / \mathrm{mL}$ of HA. The concentration of starch varied from 40 to $420 \mathrm{mg} / \mathrm{mL}$, but after preliminary evaluations (results not shown), 100 and $200 \mathrm{mg} / \mathrm{ml}$ of starch were chosen for this study. For simplicity, the sponges prepared will be identified as: 1:0:0, 1:4:0, 1:4:5 and 1:4:10 (collagen:HA:starch, by weight) from this point forward, which means the times that collagen concentration $(20 \mathrm{mg} / \mathrm{mL})$ was doubled with $\mathrm{HA}$ and/or starch. Therefore, while collagen and HA concentrations were fixed for all the sponges, the mass of starch was increased but, sponges with form of cylinder of $1 \mathrm{~cm}$ high $\times 0.5 \mathrm{~cm}$ diameter were obtained for all of the collagen:HA:starch samples, thus compression tests would be performed under the same conditions, and elastic modulus data would be attributed to the addition of HAstarch blends. Sponges were obtained by adding a solution of well mixed collagen, HA and starch into a 96 well plate (Corning, 3526), and then freeze-drying (Labconco Freeze zone, 1L) for $24 \mathrm{~h}$. Each sponge was taken out from the 96 well plate and then placed into aluminum foil trays. Sponges were cross-linked by heat-dehydration (NAPCO vacuum oven) at $145^{\circ} \mathrm{C}$ and $100 \mathrm{kPa}$ for $48 \mathrm{~h}$, which consists on removal of water from proteins to form interchain cross-links as a result of condensation reactions either by esterification or amide formation. Amino acid residues such as aspartic acid, glutamic acid, serine, threonine, arginine and lysine, which constitute $\sim 745$ of the 3156 residues on collagen, may be involved in those condensation reactions (Yannas \& Tobolsky, 1967; Weadock, Olson \& Silver, 1983-84).

\subsection{Sponge microstructure}

The surface structure of the sponges was imaged using scanning electron microscopy (SEM), before and after cell proliferation assays. The seeded sponges were washed three times with 1X PBS to completely remove the culture medium. Sponges were then stored at $4{ }^{\circ} \mathrm{C}$ with $1 \mathrm{~mL}$ of $4 \%$ formaldehyde diluted in 1X PBS, until use. Before SEM imaging, sponges were dehydrated with a graded series of ethanol, and then subjected to critical point drying to avoid shrinkage. Samples transverse sections were coated with iridium using an Emitech K575X ion sputter coater (Quorum Technologies Ltd, West Sussex, UK) and then imaged by SEM (FEI$\mathrm{XL30}$, FEI Company, Oregon, US) at a voltage of $3 \mathrm{kV}$.

\subsection{Compression testing}

Compression tests were performed to all of the fabricated sponges and CollaPlug ${ }^{\circledR}$ (each in triplicate). Samples were rehydrated in $1 \mathrm{X}$ HBSS for $0.5 \mathrm{~h}$ at room temperature prior to testing 
and were kept soaked into $1 \mathrm{X}$ HBSS during loading. Sponges were loaded at a strain rate of $1 \times 10^{-3} \mathrm{~s}^{-1}$ to $75 \%$ strain using a universal testing machine with a $500 \mathrm{~N}$ load cell (3342 Single Column System, Instron, Massachusetts, US). The small displacements were measured using an external deflectometer SATEC model 13540 (Epsilon Technology Corp., Wyoming, US). Elastic modulus was calculated from the slope of the stress-strain curve in the linear region over a $2 \%$ strain range within all data between 0 and $10 \%$ strain.

\subsection{Cell attachment assay}

\subsubsection{Cell culture}

Mouse fibroblast L929 cells were cultured in a cell culture dish with RPMI 1640 medium supplemented with $10 \%$ fetal bovine serum (FBS) and $1 \mathrm{X}$ of antibiotic/antimycotic solution in humidified atmosphere, $5 \% \mathrm{CO}_{2}$ air, at $37^{\circ} \mathrm{C}$, until confluence was $80 \%$. The cells were detached from the plate using $1 \mathrm{~mL}$ of trypsin/EDTA, and then incubated for $3 \mathrm{~min}$ at $37^{\circ} \mathrm{C}$. The cells solution obtained was centrifuged at $3000 \mathrm{~g}$ for $5 \mathrm{~min}$. After this, cells were counted using trypan blue and a TC10 Automater Cell Counter (BIORAD, 145-0001), resulting in 99\% of live cells.

\subsubsection{Cell seeding}

CollaPlug ${ }^{\circledR}$ collagen sponges were used as a positive control for all of the experiments. Sponges were sterilized by $254 \mathrm{~nm}$ UV (AB) lamp for $10 \mathrm{~min}$ in a laminar flux chamber (Thermo Scientific Forma Series II, 3110) and placed, in triplicate, in a 24 well culture plate (Corning, 3526). Sponges were soaked three times into $0.1 \mathrm{~mL}$ of RPMI supplemented medium (10\% FBS and $1 \mathrm{X}$ antibiotic-antimycotic), with $1 \times 10^{5}$ cells per well. The culture plate was incubated in humidified, $5 \% \mathrm{CO}_{2}$ air atmosphere at $37^{\circ} \mathrm{C}$. Next, the sponges were washed three times with sterile 1X PBS, and then cut into two pieces (one for cell proliferation assays, and one for SEM imaging). The scaffolds were evaluated after 2 and 5 days of contact with cells. The RPMI supplemented medium was refreshed every $48 \mathrm{~h}$.

\subsubsection{Cell viability}

After each incubation time ( 2 and 5 days), the sponges were washed three times with sterile 1X PBS to completely remove the culture medium. Afterwards, the sponges were transferred to a new 24 well culture plate. Sponges were then treated with $1 \mathrm{~mL}$ of $0.25 \%$ trypsin/EDTA for 3 min in a humidified, $5 \% \mathrm{CO}_{2}$ air atmosphere at $37^{\circ} \mathrm{C}$. After incubation, the sponges were washed with trypsin/EDTA solution and transferred to a $15 \mathrm{~mL}$ tube with $3 \mathrm{~mL}$ of fresh RPMI 
supplemented culture medium, prior centrifugation for $5 \mathrm{~min}$ at $3000 \mathrm{~g}$. Each pellet was resuspended in fresh $0.1 \mathrm{~mL}$ of RPMI supplemented medium and transferred to a new 96 well plate. Finally, $0.020 \mathrm{~mL}$ of Cell Titer 96 Aqueous One Solution (MTS assay) was added per well before incubation in a humidified, $5 \% \mathrm{CO}_{2}$ air atmosphere for $2 \mathrm{~h}$. The absorbance was read in an ELISA plate reader (BioTek, Epoch) at $495 \mathrm{~nm}$. Results were presented as the percentage of viable cells normalized with respect to the commercial control CollaPlug®, since one of the goals of this work was to investigate and compare the mechanical and in vitro properties of collagen:HA:starch sponges with respect to a commercially available collagen sponge used in dentistry, as the sponges prepared in this study are intended to be used for dental applications.

The amount of absorbance is directly proportional to the number of living cells in culture attached to the sponges; therefore sponges that showed higher absorbance were chosen for blood-clotting assay.

\subsection{Blood-clotting assay}

Blood-clotting and preservation of erythrocytes integrity capability of the collagen:HA:starch sponges were analyzed and compared with commercially available CollaPlug $®$. Blood samples were obtained from healthy volunteers by venipuncture under aseptic technique, and collected in acid citrate dextrose (ACD) anticoagulant vacutainer tubes. Sponges were cut to obtain pieces of $0.5 \mathrm{~cm}$ high $\times 0.5 \mathrm{~cm}$ diameter and placed in a 12 well plate. Clotting reaction started when $0.85 \mathrm{~mL}$ of $0.2 \mathrm{M} \mathrm{CaCl}_{2}$ was added to the total of blood collected. The activated blood $(0.1$ $\mathrm{mL}$ ) was carefully infiltrated twice through the sponges. The samples were then incubated, in triplicate, at room temperature for 5 and $15 \mathrm{~min}$, and then were incubated for $5 \mathrm{~min}$ with $3 \mathrm{~mL}$ of distillated water. Samples were centrifuged at $300 \mathrm{~g}$ during $5 \mathrm{~min}$ and $0.2 \mathrm{~mL}$ of supernatant from each sample, were transferred to a 96 well plate. The red blood cells that cannot be trapped into thrombi would be lysed. Hemoglobin released into water was read at $540 \mathrm{~nm}$ (BioTek, Epoch) using water as control as previously described (Huang et al., 2003; Sudheesh Kumar et al., 2010; Sun et al., 2009).

\subsection{Analysis of data}

Statistical analysis of results (elastic modulus, cell attachment and blood-clotting) was done by an analysis of variance (ANOVA) of the main effects. Statistical significance between each of the immobilization conditions was determined by Tukey's a posteriori test, and significance was determined at $p<0.05$. Origin Pro 8.5, Statistics 8 and Microsoft Excel were used for processing of data and statistical analysis of results. 


\section{Results and discussion}

\subsection{Structural characteristics}

Every gram of untreated ground bovine cortical bone yielded $0.125 \pm 0.017 \mathrm{~g}$ of partially soluble collagen flakes after nine days of EDTA demineralization (Fig. 2a) but completely soluble collagen $(0.028 \pm 0.001 \mathrm{~g})$ was obtained only after concentrating, by freeze-drying, the acetic acid-solubilized fraction of the collagen flakes (Fig. 2b). Therefore, collagen was extracted with a yield of $2.8 \%$ on the basis of the dry weight, a considerably good yield when taking into account, that $1.6 \%$ (wet weight) of yield was obtained when collagen was extracted from bone of bigeye snapper (Priacanthus tayenus) (Kittiphattanabawon, Benjakul, Visessanguan, Nagai \& Tanaka, 2005).

a

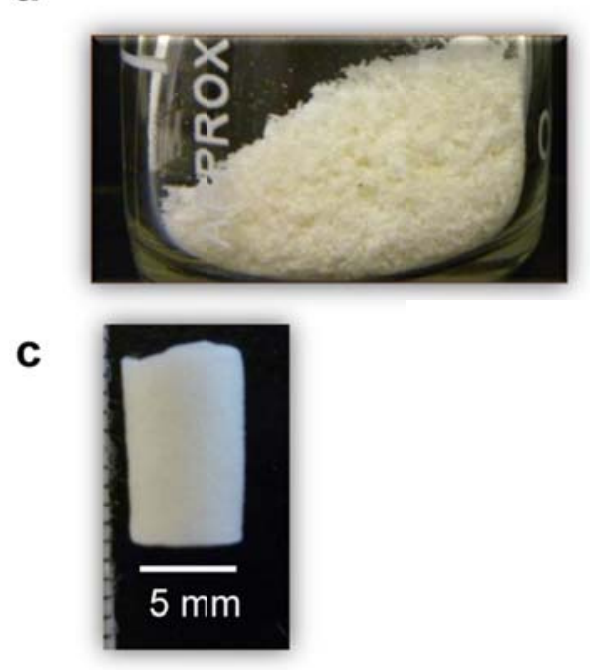

b

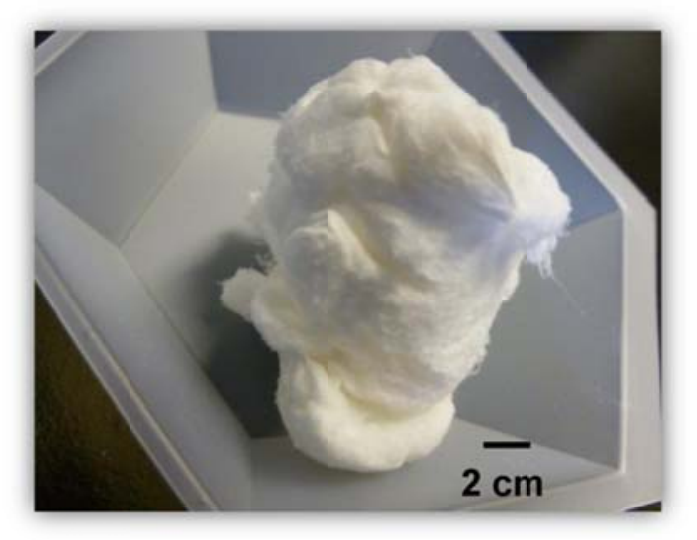

Fig. 2. Photographs showing (a) flakes of partially soluble collagen obtained by EDTA demineralization of bovine femur bone and, (b) completely soluble collagen obtained after freeze-drying the acetic acid-solubilized fraction of the collagen flakes obtained in (a). (c) This collagen was used to fabricate the collagen:HA:starch biocomposites.

The acetic acid-solubilized collagen obtained was used to prepare the collagen:HA:starch biocomposites (Fig. 2c). The morphologies of collagen sponges with different concentrations of HA and starch was analyzed by SEM (Fig. 3). SEM micrographs of the transversal section of the sponges showed that morphology changed with concentration of incorporated starch, as previously shown for sponges prepared with starch (Bice et al., 1944). Although SEM does not provide quantitative information about porosity, it can be seen that sponges 1:0:0 and 1:4:0 
(collagen:HA:starch by weight), exhibited semispherical, irregular and interconnected pores between 150 and $200 \mu \mathrm{m}$, similar to those found in CollaPlug® (Figs. 3a-c); while pore size reduction $\sim 75$ and $\sim 25 \mu \mathrm{m}$ occurred in sponges $1: 4: 5$ and 1:4:10, respectively, as starch concentration increased from 100 to $200 \mathrm{mg} / \mathrm{mL}$ (Figs. 3d-e). These sponges had a roughergranular microstructure corresponding to starch, since is apparent that concentration of the granules increased with starch concentration. In addition, the granules in the sponges had a size of $\sim 15 \mu \mathrm{m}$ like native (unmodified) starch granules (Cho \& Lee, 2002). One of the goals of this work was to improve the elastic modulus of collagen sponges, which can be achieved by chemically modifying the starch used, since its mechanical properties are weak. However, the prepared sponges should keep being compressible. To accomplish this, HA blended with native, chemically unmodified, starch granules was used, producing the granular microstructure of the starch containing sponges (Figs. 3d-e).
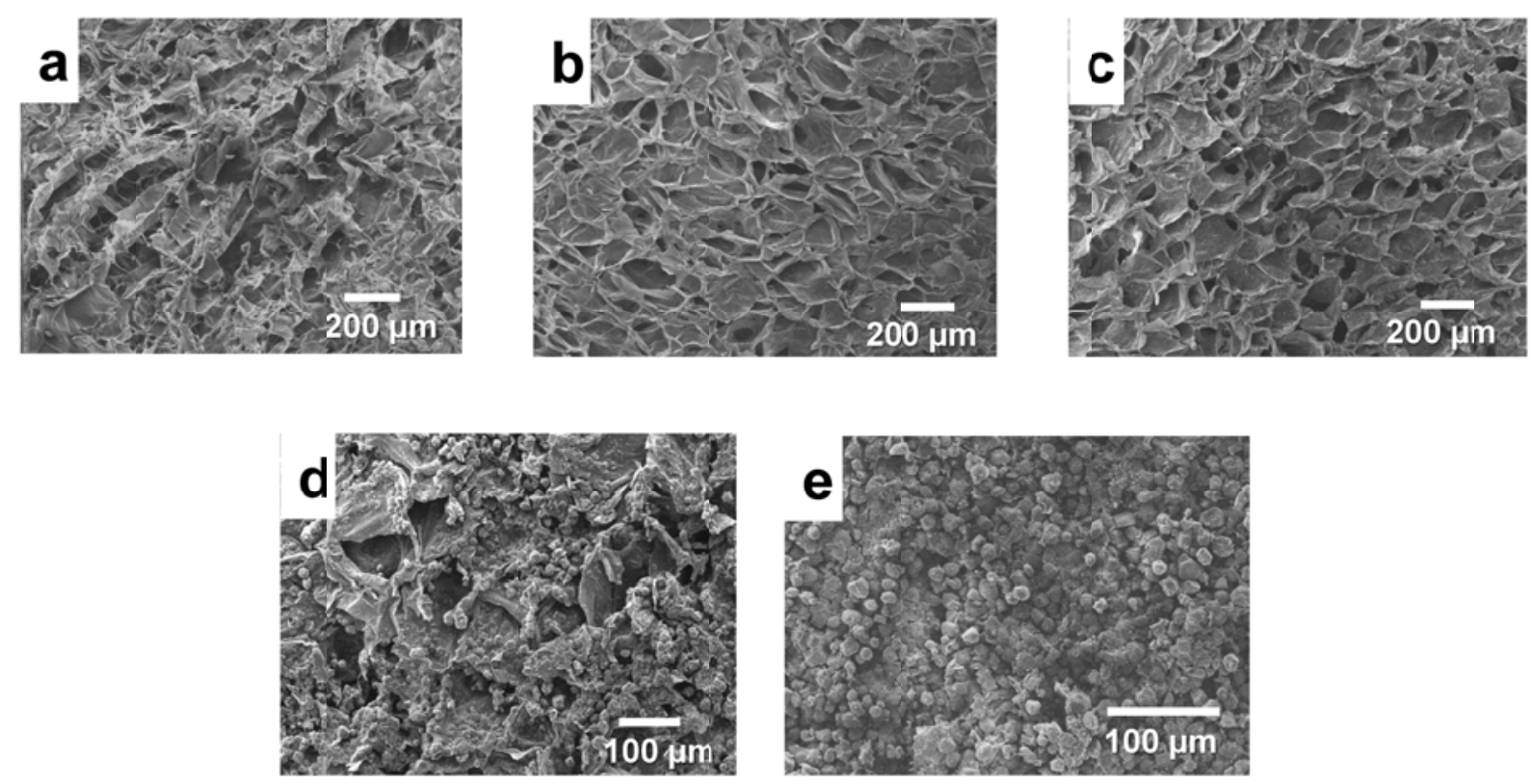

Fig. 3. Representative SEM micrographs showing the transversal section of the collagen:HA:starch sponge. (a) CollaPlug ${ }^{\circledR}$, used as commercial control, (b) 1:0:0, (c) 1:4:0, (d) $1: 4: 5$, (e) 1:4:10 collagen:HA:starch by weight. The structural characteristics of the sponges changed as starch was incorporated into the biocomposites. Semispherical and interconnected pores are visible in sponges 1:0:0 and 1:4:0 collagen:HA:starch by weight, similar to those found in CollaPlug ${ }^{\circledR}$. Pore size reduction was observed as concentration of starch increased in sponges. 


\subsection{Compression behavior of the sponges}

Similar to all of the biocomposites, collagen sponges with HA and starch exhibited compressive stress-strain curves characteristic of elastic foams (Fig. 4a) however, differences such as collagen source, addition of HA or concentration of starch, led to structural characteristics that directly affected the mechanical response of the sponges. CollaPlug®, the commercial control, had an elastic modulus of $1.45 \pm 0.20 \mathrm{kPa}$, while sponge 1:0:0 had a value of $18.30 \pm 2.00 \mathrm{kPa}$, i.e. 12.5 times higher $(P<0.05)$ than that of the commercial control. CollaPlug $®$ is made of type I collagen extracted from bovine Achilles tendon (Gruber et al., 2004), while sponges 1:0:0 (collagen:HA:starch, by weight) were prepared with collagen isolated from bovine bone (section 2.2). The collagen isolated in this work did not contain mineral after treatment with EDTA (Fig. 1a), then the increased elastic modulus can be attributed to factors other than remaining mineral. Previous studies on type I collagens isolated from different sources of the same organism, for example skin and bone from bigeye snapper, have showed that although both are type I collagens, there are slight differences on their enzymatically digested peptide maps, indicating differences in amino acid composition, therefore in their structural conformation (Kittiphattanabawon, Benjakul, Visessanguan Nagai \& Tanaka, 2005). Moreover, is been proposed that modification and rearrangement of a few amino acid motifs on spider silk proteins, directly correspond to the mechanical properties exhibited by those proteins (Hayashi, Shipley \& Lewis, 1999). Based on this, it is plausible to attribute the difference in the elastic modulus between CollaPlug $®$ and sponges 1:0:0 (collagen:HA:starch) to the different sources of collagen, i.e. tendon or bone, both from bovine. The incorporation of HA also influenced the compression behavior, as expected since HA increases the stiffness and hardness (Kane \& Roeder, 2012). The addition of HA increased the elastic modulus from $18.30 \pm 2 \mathrm{kPa}$ to $23.04 \pm$ $0.5 \mathrm{kPa}$ for 1:0:0 and 1:4:0 (collagen:HA:starch), respectively $(P<0.05)$ (Fig. 4b). However, the best elastic moduli were obtained when starch was added to the biocomposites, $31.32 \pm 2$ and $48.76 \pm 8 \mathrm{kPa}$ for sponges 1:4:5 and 1:4:10 (collagen:HA:starch, by weight) respectively. Thus elastic modulus of 1:4:10 (collagen:HA:starch) sponges was found to be two times higher with respect to $1: 4: 0$ ones $(P<0.05)$. HA adheres strongly to collagen and starch because of the polar nature of these polymers. In this case, collagen provided a framework to the sponges, while starch, the polymer present in mayor amount, could act like a dispersing agent, leading to a more homogeneous distribution of the HA through the sponges, which improves its mechanical properties. However, the decreased porosity (increased density) and the reduction of pore sizes with increasing of starch concentration (Figs. $3 \mathrm{~d}, \mathrm{e}$ ), were the main reasons for the elastic modulus increment. Thus, porosity, pore size and internal structure of sponges are the 
main factors that directly affect the mechanical performance of these materials. The relative elastic modulus $\left(E^{*} / E_{s}\right)$ and relative compressive strength $\left(\sigma^{*} / \sigma_{s}\right)$ are directly related to the fraction of porosity $(\phi)$ in a porous material (Gibson \& Ashby, 1997),

$$
\frac{E^{*}}{E_{s}} \approx(1-\phi)^{n}
$$

where $E^{*}$ is the measured elastic modulus of the porous material, $E_{s}$ is the elastic modulus of the completely dense solid and $n$ usually ranges between 1-3. This indicates that the elastic modulus is strongly correlated with the amount of porosity in the porous material.

a

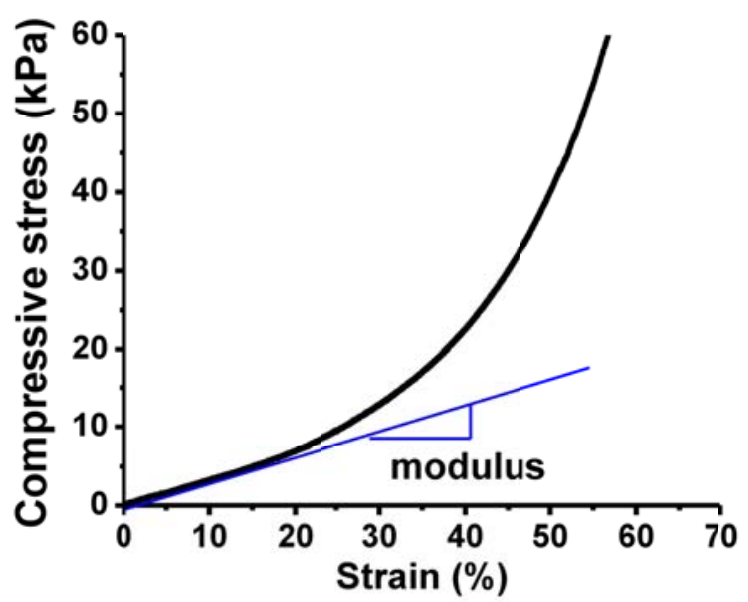

b

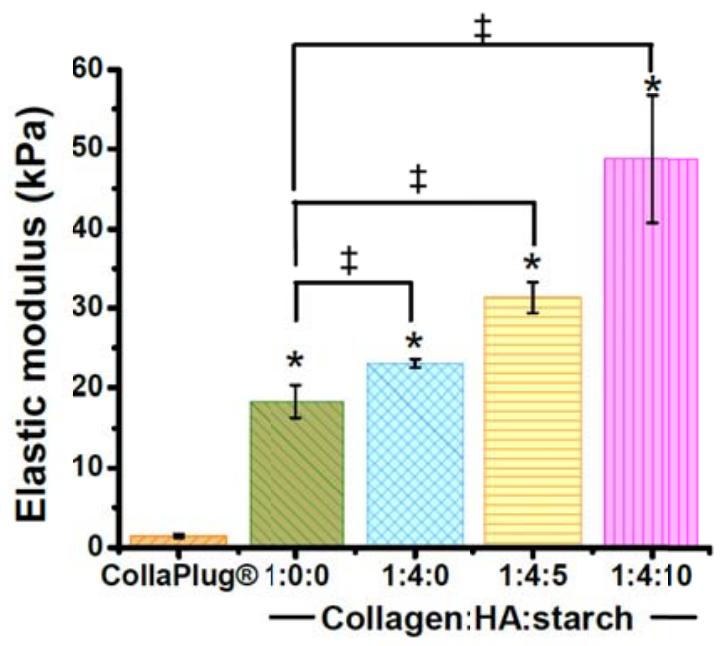

Fig. 4. Compression behavior of the sponges synthesized. (a) Representative stress-strain curve for the compression of collagen:HA:starch sponges at 1:4:5 collagen:HA:starch by weight. The elastic modulus was calculated as the slope over a $2 \%$ strain range within all data between 0 and $10 \%$ strain. (b) Mean elastic modulus of the collagen sponges reinforced with $\mathrm{HA}$ and starch at 1:0:0, 1:4:0, 1:4:5 and 1:4:10 collagen:HA:starch by weight, while CollaPlug® was used as a control ( $n=5 /$ group). Error bars show one standard deviation. Sponges prepared with the isolated collagen from bovine bone alone (sponges 1:0:0) significantly increased elastic modulus compared with CollaPlug ${ }^{\circledR}\left({ }^{*} P<0.05\right.$, sponge 1:0:0 $>$ CollaPlug ${ }^{\circledR}$, Tukey's HSD). Starch improved the elastic modulus of the sponges compared with using only HA $(P<0.05$, sponges 1:4:5 and 1:4:10 > sponges 1:4:0, Tukey's HSD). All of the compression tests were performed under wet condition (1X HBSS).

\subsection{Cell viability}

After the results obtained from the compression tests, analysis of cell viability was done on the sponges that contained the blended starch, HA and collagen, and the results were 
compared with CollaPlug ${ }^{\circledR}$ (as commercial control) which is pure collagen, and our collagen sponge (scaffold 1:0:0, collagen:HA:starch, by weight). Thus, mouse fibroblasts L929 cells were put into contact with the scaffolds and incubated for 2 and 5 days. The MTS assay was used to measure cell viability, which is a colorimetric assay that indirectly measures the metabolic activity of living cells based on the reduction of MTS reagent to brown formazan product when incubated with viable cells (Tuzlakoglu et al., 2005). Therefore, the amount of the absorbance read was directly proportional to fibroblasts cells that kept viable on the sponges. A culturegrade polystyrene well plate is generally used as positive control in the MTS assay. However, the results obtained here were normalized with respect to CollaPlug ${ }^{\circledR}$. A sponge (3D structure) resembles biological models more than $2 \mathrm{D}$ structures, i.e. the polystyrene well plate. This allowed comparison of our sponges with structures that are more similar to those found in nature. Moreover, the culture-grade polystyrene well plate promotes a higher cell growth rate compared with 3D structures, because of factors such as distribution of nutrients, which is different in the polystyrene plate monolayer and the 3D sponge. Although collagen may have some degree of cytotoxicity because of some physicochemical phenomena of degrading collagen-based material are suspected to be a cause of oxidative stress (Yamada et al., 2010), our results demonstrate that other factors such as the microstructure of the scaffolds directly influence the viability of the cells seeded on the sponges. In this case, the higher absorbance read, interpreted as higher number of cells attached to the scaffolds, was measured in CollaPlug ${ }^{\circledR}$ compared with sponge 1:0:0 $(P<0.05)$ at day 5 (Fig. 5a). Both sponges are made of type I collagen, and then this difference on cell attachment can be attributed to the structural characteristics of the scaffolds. Analysis of the microtopography of the sponges showed that surface of CollaPlug ${ }^{\circledR}$ was rougher than that of sponge 1:0:0 (collagen:HA:starch, by weight) (Figs. 3a-b). A rough surface is been previously suggested to provide sites for protein adhesion and cell attachment (Kane \& Roeder, 2012), which could be the reason for the higher cell viability found in CollaPlug ${ }^{\circledR}$ compared with our collagen sponge. However, the highest content of cells attached to sponges was found for those that contained starch in addition to collagen and $\mathrm{HA}$ at day 5 of incubation, i.e. sponges $1: 4: 5$ and $1: 4: 10$ compared with CollaPlug $®$ and sponges 1:0:0 (collagen:HA:starch) $(P<0.05)$ (Fig. 5a). The microtopography of these scaffolds showed that sponges 1:4:5 and 1:4:10 were rougher because of the granules of the incorporated starch (Figs. 3d-e), which directly influenced the cell viability by increasing the content of fibroblasts on the scaffolds. Moreover, fibroblasts on collagen:HA:starch sponges had a flattened morphology (Fig. 5b), indicating good contact between fibroblasts and sponges, directly related to the biocompatibility of the scaffolds (Nath, Tripathi \& Basu, 2009). Although 
there is no evidence so far supporting that starch per se stimulates cell viability, our results indicate that increased cell viability is attributed to starch, since collagen and HA concentrations were kept constant in all the sponges, while cell viability increased on those containing starch, which can be attributed to its granular microstructure. However, more analyses need to be done to clarify the factors that contributed to the increased cell viability.

It was found that the incorporation of starch to the sponges increased the cell viability. Although, as dental fillers, these materials should prevent volume reduction after tooth extraction, an ideal graft material placed in extraction sockets should also promote healing, i.e. bone formation, to allow initiation of osteogenesis (Serino, Rao, lezzi \& Pattelli, 2008).
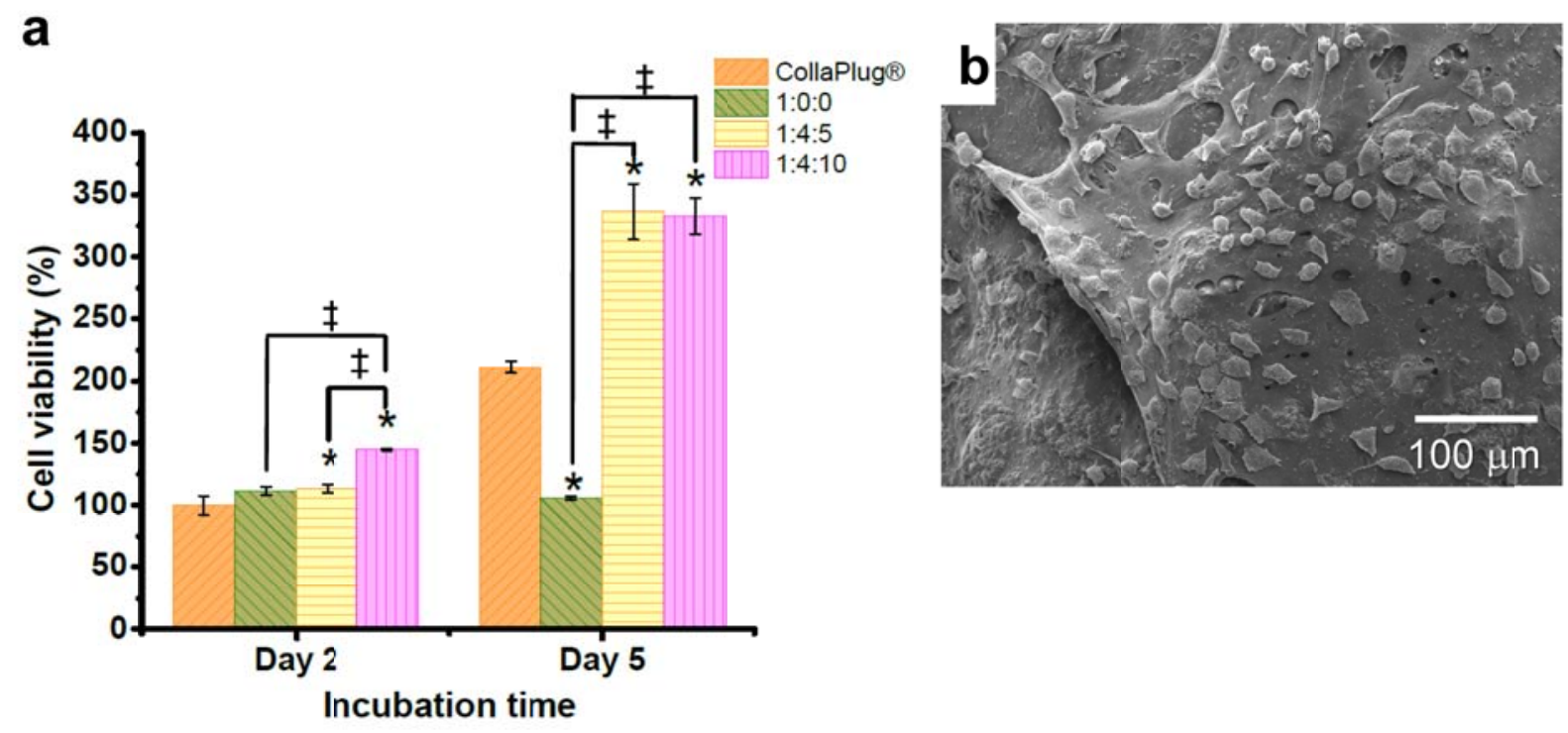

Fig. 5. Viability of fibroblast L929 cells on sponges. (a) Cell viability (MTS assay) of mouse fibroblast L929 cells cultured in collagen:HA:starch sponges at days 2 and 5 ( $n=3 /$ group). Error bars show one standard deviation of the mean. Incorporation of starch into the sponges increased cell viability at days 2 and 5 compared with those seeded on CollaPlug $\left({ }^{*} P<0.05\right.$, sponges 1:4:5 and 1:4:10> CollaPlug ${ }^{\circledR}$, Tukey's HSD) and cells seeded on pure collagen sponge ( $P<0.05$, sponges 1:4:5 and 1:4:10 > 1:0:0, Tukey's HSD). (b) Representative SEM micrograph showing fibroblast $L 929$ cells attached to sponge 1:4:10 collagen:HA:starch by weight.

\subsection{Blood-clotting}

From cell proliferation results, it was decided to test sponges 1:4:5 and 1:4:10 (collagen:HA:starch, by weight) because of the high cell proliferation obtained, and compare to the results for the commercial CollaPlug $\AA$. The blood-clotting assay performed measures the 
hemolyzed red blood cells that were not trapped in the clot formed on the sponges (Sudheesh Kumar et al., 2010), therefore the higher the absorbance read, the lower the clotting efficiency of the scaffold in terms of clotting rate. The results showed that during the first 5 min of contact with blood, CollaPlug ${ }^{\circledR}$ clotted blood more rapidly than sponges with composition 1:4:5 and 1:4:10 $(P<0.05)$ (Fig. 6). We expected that combination of two hemostatic agents with high efficacy, collagen and starch, would result in an improved clotting rate when compared with pure collagen sponges. However, two factors should be consider: 1) that sponge with composition $1: 4: 5$ contained starch with concentration 5 times the concentration of collagen, therefore it is reasonable that starch would direct the blood-clotting response of the scaffolds and, 2) that collagen and starch promote blood-clotting in different ways. While collagen is a protein that participates in platelet activation after injury by supporting the adhesion, spread and activation of platelets to form an aggregate (Farndale, Sixma, Barnes \& Groot, 2004); the granules of starch act as molecular sieves that locally dehydrate blood by absorbing its fluids and molecular components, concentrating platelets and other serum proteins on the surface of the scaffolds, which activates the clotting process (Ereth et al., 2008). Therefore, CollaPlug ${ }^{\circledR}$ (pure collagen) initiated blood-clotting more rapidly than starch, i.e. sponges 1:4:5 (collagen:HA:starch, by weight). But the blood-clotting response of the sponges was improved when starch was doubled from 5 to 10 , i.e. sponge 1:4:10 clotted more blood than sponge with composition 1:4:5 $(P<$ $0.05)$ after $5 \mathrm{~min}$ of contact with blood $(P<0.05)$ (Fig. 6). These results indicate that bloodclotting depended on starch concentration. After $15 \mathrm{~min}$ of incubation with blood, sponges $1: 4: 10$ (collagen:HA:starch, by weight) released less hemoglobin to the medium (lower absorbance) compared with CollaPlug ${ }^{\circledR}$ and sponges with composition 1:4:5 (Fig. 6). Moreover, the hemoglobin released by CollaPlug ${ }^{\circledR}$ was doubled from 5 to $15 \mathrm{~min}$ of incubation with blood, while the hemoglobin released by sponges with composition 1:4:10 was from 0.35 at 5 min to 0.39 units of absorbance at $15 \mathrm{~min}$ of incubation, i.e. a minimum change from 5 to 15 min after exposure with blood compared with CollaPlug® (Fig. 6). We speculate that over time CollaPlug ${ }^{\circledR}$ got saturated with blood, not allowing the formation of more clots, and then the membrane of more erythrocytes was disrupted and hemoglobin was released because there was no more substrate to form a clot, which was measured as an increased absorbance from 5 to 15 min of incubation. However, sponges 1:4:10 (collagen:HA:starch, by weight) absorbed blood more slowly than CollaPlug® during the first 5 min of incubation, and after 15 min the sponge 1:4:10 was not totally saturated, allowing the formation of more clots, resulting in less hemoglobin released compared with CollaPlug®. 


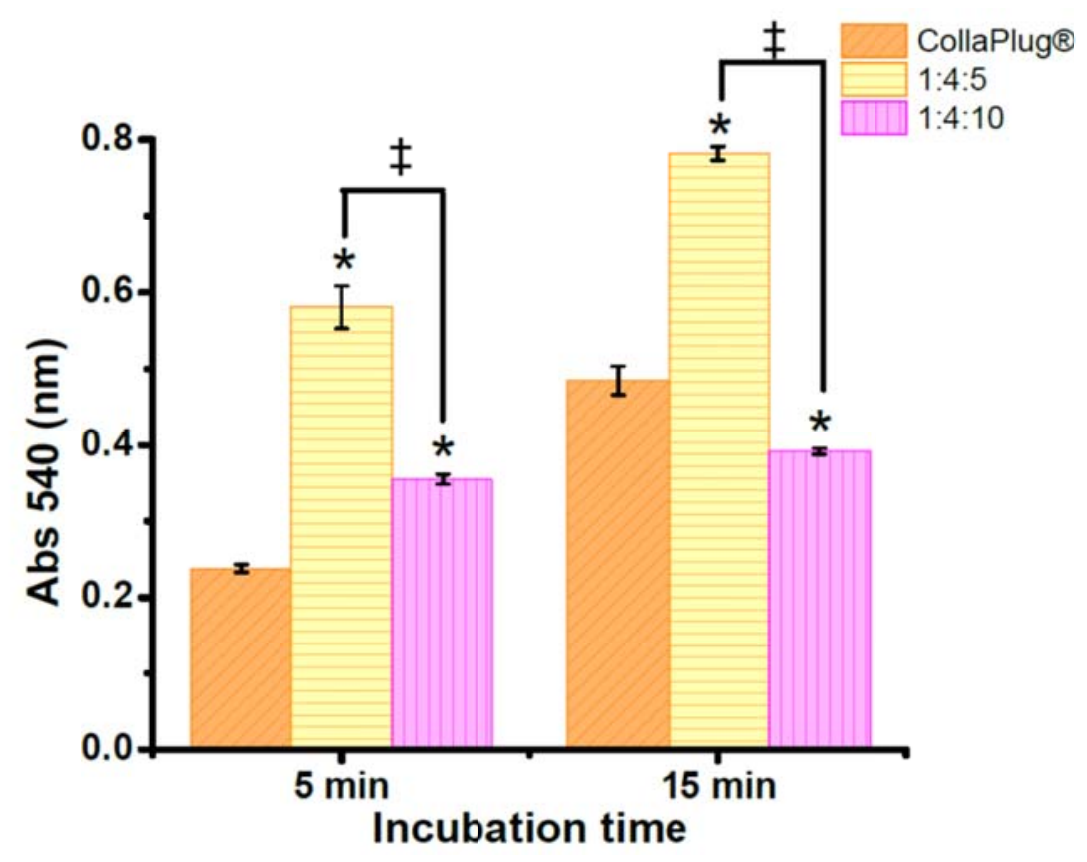

Fig. 6. Blood-clotting results for the collagen:HA:starch sponges after 5 and $15 \mathrm{~min}$ of incubation in blood ( $n=3 /$ group). Error bars show one standard deviation of the mean. CollaPlug ${ }^{\circledR}$ clotted blood more quickly than the synthesized sponges during the first 5 min of incubation in blood $\left({ }^{*} P<0.05\right.$, CollaPlug ${ }^{\circledR}>$ sponges $1: 4: 5$ and 1:4:10, Tukey's HSD). However, after 15 min of contact with blood, sponges 1:4:10 (collagen:HA:starch by weight) showed higher blood-clotting capability, i.e. lower absorbance, compared with CollaPlug ${ }^{*} P<$ 0.05 , sponge 1:4:10> CollaPlug ${ }^{\circledR}$, Tukey's HSD). Sponges with composition 1:4:10 showed better blood-clotting characteristics than $1: 4: 5$ at any incubation time evaluated $(P<0.05$, sponge 1:4:10 > 1:4:5, Tukey's HSD).

\section{Conclusions}

Collagen:HA:starch-based sponges were synthesized and evaluated in terms of their compression, cell proliferation and blood-clotting properties. The highest elastic moduli were obtained when blended HA-starch was added to the sponges. The decreased porosity (increased density) and the reduction of pore sizes with increasing of starch concentration were the main reasons for the elastic modulus increment, thus making sponges more structurally robust.

The modified microstructure and surface area influenced the cell proliferation, as a consequence of the incorporated starch, by increasing the number of cells attached to the sponges.

Sponges 1:4:10 (collagen:HA:starch) maintained their blood-clotting capability with almost no change from 5 to 15 min after contact with blood, while the commercial collagen control, 
CollaPlug ${ }^{\circledR}$, diminished to about half its capacity to absorb blood and form clots when evaluated under the same conditions after 15 min of incubation with blood.

Based on the results obtained, incorporation of starch into the sponges in a ratio of 1:4:10 (collagen:HA:starch, by weight), not only increased the elastic modulus of the collagen-HA sponges, but also increased the cell proliferation, as well as the blood-clotting capability of the scaffolds. These characteristics make collagen:HA:starch sponges a good choice for further evaluations (such as degradation studies) to be used in dentistry.

\section{Acknowledgements}

We thank Ryan Anderson of CallT2, UCSD, for helping with SEM imaging. The University of California Institute for Mexico and the United States (UC MEXUS-CONACYT) is acknowledged for the postdoctoral fellowship provided to Ana B. Castro-Ceseña. This work has been funded by the National Science Foundation, Division of Materials Research, Ceramics Program (Grant\# DMR1006931) and the Biomaterials Program (Grant\# DMR 1507978).

The authors declare no conflict of interest. 


\section{References}

Araujo-Pires, A.C., Mendes, V.C., Ferreira-Junior, O., Perri Carvalho, P.S., Guan, L. \& Davis, J.E. (2015) Investigation of a novel PLGA/CaP scaffold in the healing of tooth extraction sockets to alveolar bone preservation in humans. Clinical Implant Dentistry and Related Research. DOI: 10.1111/cid.12326

Bice, C.W., MacMasters, M.M. \& Hilbert, G.E. (1944). Proposed used of starch sponges as internal surgical dressings absorbable by the body. Science. 100(2593), 227-228.

Castro-Ceseña, A.B., Novitskaya, E.E., Phadke, A., Varghese, S. \& McKittrick, J. (2013). Isolation of collagen from cortical bovine bone for preparation of porous collagen sponges. Mechanics of Biological Systems and Materials, Conference Proceedings of the Society for Experimental Mechanics Series. 5:73-78, DOI: 10.1007/978-1-4614-4427-5_11

Chang, S.J., Huang, Y.-T., Yang, S.-C., Kuo, S.-M. \& Lee, M.-W. (2012) In vitro properties of gellan gum sponge as the dental filling to maintain alveolar space. Carbohydrate polymers. 88, 684-689.

Cho, C.G. \& Lee, K. (2002) Preparation of starch-g-polystyrene copolymer by emulsion polymerization. Carbohydrate Polymers. 48, 125-130.

Ereth, M.H., Schaff, M., Ericson, E.F., Wetjen, N.M., Nuttall, G.A. \& Oliver, Jr., W.C. (2008) Comparative safety and efficacy on topical hemostatic agents in rat neurosurgical model. Operative Neurosurgery. 63(2), ONS369- ONS372.

Farndale, R.W., Sixma, J.J., Barnes, M.J. \& De Groot, P.G. (2004) The role of collagen in thrombosis and hemostasis. Journal of Thrombosis and Hemostasis. 2, 561-573.

Gibson, L.J. \& Ashby, M.F. (1997) Cellular Solids: Structure and Properties. (2nd ed.), Cambridge: Cambridge University Press, (Chapter 4).

Go, B.T., Teoh, L.Y., Tan, D.B.P., Zang, Z. \& Teo, S.H. (2015) Novel 3D polycaprolactone scaffold for ridge preservation - a pilot randomized controlled clinical trial. Clinical Oral Implants Research. 26, 271-277.

Gruber, H.E., Lesli,K., Ingram, J., Norton, J. \& Hanley, E.N. (2004). Cell-based tissue engineering for the intervertebral disc: in vitro studies of human disc cell gene expression and matrix production within selected cell carries. The spine journal, 4, 44-55.

Hansson, S. \& Halldin, A. (2012) Alveolar ridge resorption after tooth extraction: A consequence of a fundamental principle of bone physiology. Journal of Dental Biomechanics. 3, 1758736012456543 . doi:10.1177/1758736012456543. 
Hayashi, C.Y., Shipley, N.H. \& Lewis, R.V. (1999). Hypothesis that correlate the sequence, structure, and mechanical properties of spider silk proteins. International Journal of Biological Macromolecules. 24, 271-275.

Hiraoka, Y., Kimura, Y., Ueda, H. \& Tabata, Y. (2003) Fabrication and biocompatibility of collagen sponge reinforced with poly(glycolic acid) fiber. Tissue Engineering. 9(6), 1101-1112. Huang, N., Yang, P., Leng, Y.X., Chen, J.Y., Sun, H., Wang, J., Wang, J., Ding, P.D., Xi, T.F. \& Leng, Y. (2003). Hemocompatibility of titanium oxide films. Biomaterials. 24, 2177-2187.

Kane, R.J. \& Roeder R.K. (2012). Effects of hydroxyapatite reinforcement on the architecture and mechanical properties of freeze-dried collagen sponges. Journal of the Mechanical Behavior of Medical Materials. 7, 41-49.

Kane, R.J., Weiss-Bilka, H.E., Meagher, M.J., Liu, Y., Gargac, J.A., Niebur, G.L., Wagner, D.R. \& Roeder R.K. (2015) Hydroxyapatite reinforced collagen scaffolds with improved architecture and mechanical properties. Acta Biomaterialia. 17, 16-25.

Kim, Y.-K., Yun, P.-Y., Lee, H.-J., Ahn, J.-Y. \& Kim, S.-G. (2011) Ridge preservation of the molar extraction socket using collagen sponge and xenogeneic bone grafts. Implant Dentistry. 20(4), 267-272.

Kittiphattanabawon, P., Benjakul, S., Visessanguan, W., Nagai, T. \& Tanaka, M. (2005) Characterization of acid-soluble collagen from skin and bone of bigeye snapper (Priacanthus tayenus). Food chemistry. 89, 363-372.

Korchin, L. (1956) An investigation to determine the effects of starch sponge implanted in bone. Journal of Dental Research. 35(3), 446-457.

Leblebicioglu, B., Salas, M., Ort, Y., Jhonson, A., Yildiz, V.O., Kim, D.-G., Agarwal, S. \& Tatakis, D.N. (2013) Determinants of alveolar ridge preservation differ by anatomic location. Journal of Clinical Periodontology. 40, 387-395.

López-López, J., Estrugo-Devesa, A., Jané-Salas, E. \& Segura-Egea, J.J. (2012) Inferior alveolar nerve injury resulting from overextension of an endodontic sealer: non-surgical management using the GABA analogue pregabalin. International Endodontic Journal. 45, 98104.

Nair, L.S. \& Laurencin C.T (2006) Polymers as biomaterials for tissue engineering and controlled drug delivery. Advances in Biochemical Engineering/Biotechnology. 102, 47-90.

Nath, S., Tripathi, R. \& Basu, B. (2009) Understanding phase stability, microstructure development and biocompatibility in calcium phosphate-titania composites, synthesized from hydroxyapatite and titanium powder mix. Materials Science \& Engineering C. 29, 97-107. 
Ohno, T., Tanisaka, K., Hiraoka, Y., Ushida, T., Tamaki, T. \& Tateishi, T. (2004) Effect of type I and type II collagen sponges as 3D scaffolds for hyaline cartilage-like tissue regeneration on phenotypic control of seed chondrocytes in vitro. Materials Science \& Engineering C. 24, 407411.

Ratanavaraporn, J., Kanokpanont, S., Tabata, Y.\& Damrongsakkul, S. (2008). Effects of acid type on physical and biological properties of collagen sponges. Journal of Biomaterials Science, Polymer Edition. 19(7), 945-952.

Rodrigues, C.V.M., Serricella, P., Linhares, A.B.R., Guerdes, R.M., Borojevic, R., Rossi, M.A., Duarte, M.E.L. \& Farina, M. (2003) Characterization of a bovine collagen-hydroxyapatite composite scaffold for bone tissue engineering. Biomaterials. 24, 4987-4997.

Sadjadi, M.S., Meskinfam, M. \& Jazdarreh, H. (2010) Hydroxyapatite - starch biocomposites synthesis and characterization. International Journal of Nano Dimension. 1(1), 57-63.

Salgado, A.J., Coutinho, O.P. \& Reis, R.L. (2004) Novel starch-based scaffolds for bone tissue engineering: cytotoxicity, cell culture, and protein expression. Tissue Engineering. 10(3/4), 465-474.

Salgado, A.J., Gomes, M.E., Chou, A. Coutinho, O.P., Reis, R.L. \& Hutmacher, D.W. (2002) Preliminary study on the adhesion and proliferation of human osteoblasts on starch-based scaffolds. Materials Science \& Engineering C. 20, 27-33.

Salgado, A.J., Figueiredo, J.E., Coutonho, O.P. \& Reis, R.L. (2005) Biological response to premineralized starch based scaffolds for bone tissue engineering. Journal of Materials in Medicine. 16, 267-275.

Serino, G., Biancu, S., lezzi, G. \& Piattelli, A. (2003) Ridge preservation following tooth extraction using a polylactide and polyglycolide sponge as space filler: a clinical and histological study in humans. Clinical Oral Implant Research. 14, 651-658.

Serino, G., Rao, W., lezzi, G. \& Piatelli, A. (2008) Polylactide and polyglycolide sponge used in human extraction sockets: bone formation following 3 months after its application. Clinical Oral Implants Research. 19(1), 26-31.

Shibata, N., Nishumura, A., Naruhashi, K., Nakao, Y. \& Miura, R. (2010) Preparation and pharmaceutical evaluation of new sustained-release capsule including starch-sponge matrix (SSM). Biomedicine \& Pharmacotherapy. 64, 352-358.

Soares, E.C.S., Costa, F.W.G., Bezerra, T.P., Nogueira, C.B.P., Batista, S.H.B., de Barros Silva. P.G., Sousa, F.B. \& Sá Roriz Fonteles, C. (2015) Postoperative hemostatic efficacy of gauze soaked in tranexamic acid, fibrin sponge, and dry gauze compression following dental 
extraction in anticoagulated patients with cardiovascular disease: a prospective, randomized study. Oral and Maxillofacial Surgery. 19, 209-216.

Sudheesh Kumar, P.T., Abhilash, S., Manzoor, K., Nair, S.V., Tamura, H. \& Jayakumar, R. (2010). Preparation and characterization of novel $\beta$-chitin/nanosilver composite sponges for wound dressing applications. Carbohydrate Polymers. 80, 761-767.

Sun, Z.J., Wu, L., Huang, W., Zhang, X.L., Lu, X.L., Zheng. Y.F., Yang, B.F. \& Dong, D.L. (2009). The influence of lactic on the properties of poly(glycerol-sebacate-lactic acid). Materials Science \& Engineering C. 29, 178-182.

Taira, M., Sasaki, K., Saitoh, S., Nezu, T. \& Araki, Y. (2006) Preparation of highly poreinterconected porous collagen sponges using chloroform and ammonia. Journal of Oral Tissue Engineering. 4(2), 68-76.

Thalmair, T., Fickl, S., Schneider, D., Hinze, M. \& Wachtel, H. (2013) Dimensional alterations of extraction sites after different alveolar ridge preservation techniques - a volumetric study. Journal of Clinical Periodontology. 40, 721-727.

Tuzlakoglu, K., Bolgen, N., Salgado, A.J., Gomes, M.E., Piskin, E. \& Reiss, R.L. (2005) Nanoand micro-fiber combined scaffolds: A new architecture for bone tissue engineering. Journal of Materials Science: Materials in Medicine. 16, 1099-1104.

Vignoletti, F., Matesanz, P., Rodrigo, D., Martin, C. \& Sanz, M. (2012) Surgical protocols for ridge preservation after tooth extraction. A systematic review. Clinical Oral Implants Research. 23(5), 22-38.

Wang, R.E. \& Lang, N. (2012) Ridge preservation after tooth extraction. Clinical Oral Implants Research. 26(6), 147-156.

Wang, H.-L. \& Tsao, Y.-P. (2007) Mineralized bone allograft-plug socket augmentation: rationale and technique. Implant Dentistry. 16(1), 33-41.

Weadock, K., Olson, R.M. \& Silver, F.H. (1983-84) Evaluation of collagen crosslinking techniques. Biomaterials, Medical Devices and Artificial Organs. 11(4), 293-318.

Wei, G. \& Ma, P.X. (2004) Structure and properties of nano-hydroxyapatite/polymer composite scaffolds for bone tissue engineering. Biomaterials. 25, 4749-4757.

Yamada, M., Kubo, K., Ueno, T., Iwasa, F., Att, W., Hori, N. \& Ogawa, T. (2010) Alleviation of comercial collagen sponge- and membrane-induced apoptosis and dysfunction in cultured osteoblasts by amino acid derivative. The International Journal of Oral \& Maxillofacial Implants. 25, 939-946.

Yannas, I.V. \& Tobolsky, A.V. (1967) Cross-lining of gelatin by dehydration. Nature. 215, 509510. 
Yunoki, S., Ikoma, T., Monkawa, A., Ohta, K., Kikichi, M., Sotome, S., Shinomiya, K. \& Tanaka, J. (2006) Control of pore structure and mechanical property in hydroxyapatite/collagen composite using unidirectional ice growth. Materials Letters. 60, 999-1002.

Yunoki, S., Ikoma, T. \& Tanaka, J. (2010) Development of collagen condensation method to improve mechanical strength of tissue engineering scaffolds. Materials Characterization. 61, 907-911.

Zou, L., Liu, J., Yin, S., Li, W. \& Xie, J. (2008) In vitro evaluation of the sealing ability of MTA used for the repair of furcation perforations with and without the use of an internal matrix. Oral Surgery, Oral Medicine, Oral Pathology, Oral Radiology, and Endodontology. 105, e61-e65. 